\title{
Indicadores de desempenho de bibliotecas na Fiocruz: um caminho em construção
}

\author{
Maria Cristina S. Guimarães \\ Doutora em ciência da informação, UFRJ/Ibict. \\ E-mail: csguima@terra.com.br \\ Etiene Gonzalez Lins \\ Bibliotecária. \\ E-mail: etiene.lins@gmail.com
}

\section{Jeorgina Gentil Rodrigues}

Mestre em ciência da informação.

E-mail: jeorgina@cict.fiocruz.br

\section{Camila Clementino Lamarão}

Bolsista Faperj.

E-mail: clamarao@cict.fiocruz.br

Vânia Guerra da Silva

Bibliotecária da Fiocruz/Cict.

E-mail: vguerra@cict.fiocruz.br

Diones Ramos da Silva

Bibliotecária da Fiocruz/IFF.

E-mail:diones@cict.fiocruz.br

\section{Resumo}

A ampla e crescente inserção das tecnologias de informação e comunicação (TICs) nos serviços de informação aponta para a necessidade de adoção de novas abordagens de gestão, especialmente nas bibliotecas acadêmicas. O desafio se avoluma quando se trata do desempenho de bibliotecas em instituições complexas, que acolhem diversos espaços de informação, frutos de práticas, temas e interesses de pesquisa diferenciados. Esse é o caso do Centro de Informação Científica e Tecnológica (Cict), unidade técnica da Fundação Oswaldo Cruz (Fiocruz), instituição que ocupa papel de liderança no cenário internacional no campo da pesquisa em saúde. Partindo da Norma ISO 11620 , o presente trabalho relata o desenvolvimento e o caminho até aqui percorrido por um projeto de pesquisa que objetiva a escolha de um conjunto de indicadores de desempenho aplicáveis a três diferentes bilbiotecas especializadas do Cict.

\section{Palavras-chave}

Indicadores de desempenho. Norma ISO 11620. Indicadores de desempenho de biblioteca.

\section{Performance indicators of libraries at Fiocruz: a building-up path}

\begin{abstract}
The increasing impact of information and communication technologies (ICTs) in the traditional libraries, especially in academic ones, has point to the necessity of taking in new management approaches. The challenge is even more complex for research institutions that encompass various specialized libraries as a result of different researches interests and social practices. The lack of unified evaluation methods has undermined any attempt to introduce modern management techniques. In searching for a standard for library performance measurement, the Scientific and Technological Health Information Center - Cict/FIOCRUZ took the ISO 11620 as starting point of a research project for work out the conception, methodology and criteria of assessment of the three different specialized libraries which are under its coordination.
\end{abstract}

Keywords

Performance indictors. Norm ISO 11620. Performance indicators of libraries.

\section{INTRODUÇÃO}

Os serviços de informação e, mais especialmente as bibliotecas acadêmicas foram protagonistas de vários dos principais eventos que demarcam o nascimento e florescimento da "era da informação" e chegaram ao século XXI com ampla agenda de oportunidades e desafios. Esse novo ambiente, especialmente caracterizado por ampla inserção de tecnologias de informação e comunicação (TICs), aponta para a adoção de novas abordagens de gestão. Inovar é a palavra-chave, (re)aprender é o caminho, qualidade dos serviços é a meta.

A busca pela qualidade não se caracteriza como um novo desafio para as bibliotecas: ao contrário, é um conceito que, na atualidade, deve ser reelaborado, enriquecido, ampliado em seus sentidos para acolher um perfil cada vez mais diferenciado de usuários, além da ampliação do espectro de atores que compõem a audiência diligente que habita a circunvizinhança dos serviços de informação (steakholders). Assim, o quesito qualidade deixa de ser um atributo circunscrito à perspectiva interna das bibliotecas. As estatísticas dos serviços continuam a ocupar lugar de destaque na gestão dos serviços, indispensáveis que são aos processos de planejamento e gestão. Entretanto, as estatísticas per se não são suficientes para traduzir e explicitar o impacto dos serviços nos usuários e na instituição. $\bigcirc$ foco passa, então, para a avaliação de desempenho.

Nos últimos 30 anos e especialmente na Grã-Bretanha e nos Estados Unidos da América, o interesse pela área de avaliação levou ao desenvolvimento e divulgação de grande variedade de estudos e abordagens sobre medidas de desempenho. Nasceram daí orientações metodológicas diversas, com focos e recortes em diferentes atividades das bibliotecas, produzindo amplo conjunto de indicadores de desempenho. Essas diferentes abordagens em muito contribuíram para o entendimento dos diferentes contextos e processos de gestão de bibliotecas. Mas o que se ganhou em diversidade perdeuse em padronização. Até o começo dos anos 80 do século $\mathrm{XX}$, eram escassas as possibilidades de se efetuarem estudos comparativos entre serviços de informação.

A ausência de linguagem comum e a necessidade de padronização guiaram os esforços da International 
Standard Organization (ISO) na elaboração de padrões para medida de desempenho das bibliotecas, resultando na Norma ISO 11620/98 (Information and Documentation - Library Performance Indicators). Esse movimento em prol da padronização surgiu em um contexto mundial de globalização, de integração de blocos regionais, de disputa internacional por fundos de pesquisa e, não menos importante, de cooperação internacional entre bibliotecas.

A norma, aplicável a todo tipo de biblioteca, coloca no centro de suas definições a noção de desempenho. O conjunto de indicadores de desempenho proposto pela norma procura equacionar três conceitos fundamentais do processo de planejamento: objetivos, meios e resultados. O desempenho se apóia na relação entre eficiência (emprego dos meios) e eficácia (alcance dos objetivos). Uma medida de desempenho deve indicar se o planejado está sendo alcançado. Assim, o processo de avaliação pode ser conduzido à luz da missão, dos objetivos e das condições contextuais em que cada biblioteca está inserida, respeitando-se assim as características que a tornam única.

É exatamente a diversidade de perfil o que melhor caracteriza o conjunto de bibliotecas que respondem pelos serviços de informação na Fundação Oswaldo Cruz (Fiocruz). Instituição vinculada ao Ministério da Saúde, a Fiocruz é reconhecida pela competência internacional no campo da pesquisa, desenvolvimento, produção e formação de recursos humanos em ciências da saúde e biomédicas. Para atender a tão amplo e diversificado leque de atividades, cuja dinâmica se estende ao longo dos últimos 106 anos, a Fiocruz conta atualmente com 10 (dez) bibliotecas, distribuídas pelos seus centros regionais. Cada uma delas se insere em um contexto ímpar da prática na saúde, possui acervo diferenciado e desenvolve atividades particulares com vistas a atender a um grupo de usuários com perfil específico e necessidades próprias.

A prática da avaliação de serviços, especialmente por meio de produção de estatísticas dos serviços, ainda que reconhecida e abraçada como atividade importante para o planejamento e gestão coordenada das bibliotecas, ainda carece de uma visão orgânica e integradora. Paralelamente, em tempos de recursos financeiros escassos, eficiência e eficácia são conceitos-chave para produzir evidências para o tomador de decisão. A padronização das medidas de desempenho, tomando a ISO 11620 como ponto de partida, foi a estratégica adotada na busca de harmonia entre as métricas atuais de cada biblioteca, tendo em vista a implantação de um programa de avaliação de desempenho das bibliotecas Fiocruz.

O presente trabalho relata o caminho até aqui percorrido por um projeto de pesquisa em curso que objetiva a escolha de um conjunto de indicadores de desempenho aplicáveis às bibliotecas da Fiocruz. O projeto tem como foco inicial as três maiores bibliotecas da instituição, as quais estão sob a coordenação do Centro de Informação Científica e Tecnológica em Saúde (Cict). O texto procura ainda retratar o ambiente de cooperação e colaboração que vem propiciando uma discussão frutífera e um estratégico processo de aprendizagem.

\section{O FOCO DA PESQUISA}

A Fundação Oswaldo Cruz (Fiocruz) é uma instituição vinculada ao Ministério da Saúde e abriga atividades que incluem o desenvolvimento de pesquisas, a prestação de serviços hospitalares e ambulatoriais de referência em saúde, a fabricação de vacinas, medicamentos, reagentes e kits de diagnóstico, o ensino e a formação de recursos humanos, a informação e a comunicação em saúde, ciência e tecnologia, o controle da qualidade de produtos e serviços e a implementação de programas sociais. Tem como missão promover a saúde e o desenvolvimento social, gerar e difundir conhecimento científico e tecnológico, ser um agente da cidadania. Por meio de programas de informação e comunicação, vem procurando cumprir papel da maior relevância, o que deu margem, certamente, a que fosse premiada pela Unesco, em 2002, como a instituição do ano na área das ciências.

A Fiocruz guarda em sua história uma relação visceral com a informação. É viva, em sua memória, cultura e imaginário, a fala de seu patrono e fundador, Oswaldo Cruz, personalidade reconhecida internacionalmente: "Corte-se até a verba para a alimentação. Mas não se sacrifique a biblioteca". Atualmente, a instituição conta com dez bibliotecas distribuídas em diferentes regiões do país, cada uma atendendo a um perfil diferenciado de usuários e a suas respectivas necessidades de informação. O comprometimento com e a valorização da informação como ingrediente vital para a pesquisa é um dos fatores que certamente conduziram, nos anos 80 , à criação de uma unidade técnica dedicada à guarda, planejamento e gestão da informação científica e tecnológica no campo da saúde, o Centro de Informação Científica e Tecnológica (Cict). 
O Cict é responsável por formular políticas, desenvolver estratégias e executar ações de informação e comunicação no campo da ciência e tecnologia em saúde que respondam às demandas da sociedade e do Sistema Único de Saúde (SUS) e de outros órgãos governamentais. Atua também na coordenação da Rede de Bibliotecas da Fiocruz, que tem como principal finalidade reunir, articular e integrar as bibliotecas da instituição, visando a potencializar e agilizar o intercâmbio e o uso de informação e expandir o seu acesso e disponibilidade, para atender às necessidades e demandas de informação da comunidade científica e tecnológica em saúde, assim como à sociedade em geral (http://rede.cict.fiocruz.br/) . Ao Cict estão diretamente subordinadas três dessas bibliotecas especializadas, as quais se colocam como foco do presente estudo.

A Biblioteca de Manguinhos (Bibmang) atua como centro de referência em biomedicina para as instituições de pesquisa no país e oferece suporte ao desenvolvimento do ensino e da pesquisa no âmbito da Fiocruz. Atende ao corpo de docentes, de pesquisadores e alunos de pósgraduação da instituição e da comunidade em geral. A Biblioteca do Instituto Fernandes Figueiras (Bibiff) está voltada para saúde da mulher, da criança e do adolescente. Suas áreas de especialização são o aleitamento materno, genética, ginecologia e obstetrícia, infecção hospitalar, nutrição, patologia e pediatria. Seu público-alvo é composto por profissionais de saúde, alunos de pós-graduação, professores, pesquisadores do Instituto Fernandes Figueiras e da rede pública e privada de serviços de saúde. Por fim, a Biblioteca da Escola Nacional de Saúde Pública Sergio Arouca (Bibensp) atende ao corpo de docentes, pesquisadores e alunos de pós-graduação da Escola Nacional de Saúde Pública (Ensp), e é aberta também à comunidade em geral. Atua como centro de referência a todos os cursos descentralizados de saúde pública desenvolvidos no país e à rede pública de serviços de saúde no estado do Rio de Janeiro.

Essas bibliotecas foram criadas em momentos próprios, para atender a clientelas e necessidades particulares, e se inserem em contextos singulares de práticas do campo da saúde. A estratégia de planejamento e gestão se faz em um delicado balanço entre autonomia e coordenação central, no reconhecimento das especificidades e na responsabilidade de um todo harmônico que deve ser traduzida, de forma transparente, ao tomador de decisão, especialmente no que diz respeito aos investimentos financeiros. As atividades relacionadas à mensuração de indicadores de desempenho, ainda que realizada anualmente, carece de caráter sistêmico. Da mesma forma, a realização de estudos de satisfação de usuários ainda não está incorporada como instrumento de gestão.

Ciente de suas responsabilidades, especialmente sob a perspectiva do uso das melhores práticas de gestão, o Cict aceitou o desafio de desenvolver, de forma cooperativa junto às bibliotecas citadas, um projeto visando a uma metodologia padrão para avaliação de desempenho das mesmas. A Norma ISO 11620 se colocou então como instrumento estratégico para, em uma etapa inicial, prover uma padronização das métricas de desempenho das bibliotecas.

\section{INDICADORES DE DESEMPENHO - DEFINIÇÕES E EXPERIÊNCIAS}

O dinamismo e velocidade das transformações sociais que caracterizam a sociedade contemporânea requerem das instituições celeridade nas respostas e flexibilidade para adaptarem-se a novas e desafiantes situações. Entretanto, há sempre de se buscar sustentabilidade nas mudanças e inovações. Lancaster (1996, p. 3) ressalta que

(...) crescimento saudável implica adaptação a condições constantemente mutáveis, e adaptação implica avaliação para determinar que mudanças precisam ser feitas e qual a melhor maneira de realizálas.

A avaliação é ou deve ser o processo que gera evidências e fornece ao tomador de decisão informações que permitem justificar as ações presentes e orientações futuras. Nesse sentido, a avaliação é uma etapa necessária tanto para os gestores quanto para os profissionais de informação/bibliotecários: para os primeiros, deve ser capaz de responder pela eficácia e eficiência dos investimentos feitos, para os segundos, deve explicitar a qualidade e bom desempenho de suas atuações.

Mandani (2004) alerta para a importância da clareza conceitual quando se trata com o processo de avaliação. $\mathrm{O}$ primeiro esclarecimento deve ser feito entre medida de desempenho e avaliação. A primeira é uma medida de uma atividade ou de um serviço. Mas a medida de desempenho é um instrumento pelo qual julgamentos são feitos no curso de um processo de avaliação. O desempenho, por si só, não é bom ou ruim, positivo ou negativo, ele é o que é. Somente diante de objetivos propostos, de metas a serem alcançadas, é que um desempenho pode ser aferido. Assim, qualquer avaliação depende de um 
objetivo e de uma medida de desempenho. $\mathrm{O}$ indicador, finalmente, é uma expressão (numérica, simbólica ou semântica) que caracteriza atividades (objetos, pessoas, eventos) quantitativa ou qualitativamente, com vistas a aferir o valor dessas atividades. Qualidade das fontes de informação, relação terminais de consulta/número de usuários e satisfação dos usuários são exemplos de indicadores usados em avaliações de serviço de informação.

Em artigo recente, Rozados (2005) faz uma discussão e síntese do uso de indicadores na gestão de recursos de informação. Indicadores de input (entrada), output (saída) e outcomes (resultados) formam a tipologia mais conhecida de indicadores e expressam a visão sistêmica de uma biblioteca. Dos três, os indicadores de resultados representam os melhores esforços para aferir a qualidade e o impacto dos serviços prestados e colocam o usuário no centro de atenção das metodologias de avaliação.

A avaliação de desempenho, em contrapartida, engloba a coleção e análise de dados estatísticos para retratar o desempenho de uma biblioteca, ou seja, uma medida de comparação entre o que está sendo feito (desempenho), com o que deveria ser feito (missão) e com aquilo que se gostaria de fazer (metas). O foco na avaliação de desempenho é principalmente resultado da integração de blocos regionais no contexto internacional, especialmente na Europa, de onde nasceu do desafio de implementar metodologia única para avaliar as bibliotecas nacionais, tão peculiares que são na guarda e conservação do patrimônio nacional. Entrou em campo a ISO com a Norma ISO 11620, publicada em 1998, que congrega um conjunto de definições, metodologias e a definição de 29 indicadores de desempenho associados a um ou mais métodos de obtenção de dados e análise de resultados. Em 2003, foi publicada uma atualização da referida norma (ISO11620:1998/Adm.1/2003), com a incorporação de novos indicadores de desempenho.

Como instrumento de padronização, a ISO 11620 estabelece um grupo de indicadores para aplicação em todos os tipos de bibliotecas. Além disso, estabelece uma relação entre qualidade de serviços de biblioteca e o tópico mais amplo de administração e garantia de qualidade, uma vez que valida e apóia-se na Norma ISO 9004-2 (INTERNATIONAL ORGANIZATION FOR STANDARDIZATION, 1991). Para cada indicador, constam objetivo, escopo, definição, metodologia, interpretação e fontes de referência, e, entre os procedimentos, são adotados cálculo de percentual, amostragem, questionário e entrevista. A norma oferece também detalhada orientação sobre a metodologia e análises sobre seu emprego. Alguns serviços e atividades importantes, como, por exemplo, treinamento de usuário e serviços eletrônicos, não estão contemplados, mas são cobertos em outra norma, a ISO 2789/2003 (INTERNATIONAL ORGANIZATION FOR STANDARDIZATION, 2003b).

Muitas iniciativas de desenvolvimento de metodologias de avaliação foram empreendidas nos países desenvolvidos nos últimos 30 anos, muitas delas já englobando os serviços eletrônicos. Algumas das mais importantes são citadas:

- O projeto LibEcon nasceu na Comunidade Européia e congrega atualmente 37 países também da América, Ásia e Austrália. Os dados são coletados de acordo com a ISO 2789 e ISO 11620, possibilitando a prática de benchmarking.

- O projeto EQUINOX - Library Performance Measurement and Quality Management System nasceu com o foco em serviços eletrônicos. São 14 indicadores de desempenho acordados entre bibliotecários de seis países europeus, indicadores esses que se somam à Norma ISO11620.

- LibQUAL $+{ }^{\mathrm{TM}}$ (Libqual $+{ }^{\mathrm{TM}}$, 2005), iniciativa norteamericana resultado de uma associação entre a Association of Research Libraries (ARL) e a Texas A\&M University (TAMU), é um instrumento de aferição da qualidade dos serviços em função das percepções e expectativas dos usuários. LibQUAL $+{ }^{\mathrm{TM}}$ foi desenvolvido a partir do SERVQUAL, reconhecido como primeiro padrão desenvolvido para avaliação da qualidade de serviço no setor comercial, trabalho pioneiro de Berry, Parasuraman e Zeithaml (PARASURAMAN e outros, 1985), posteriormente adaptado para aplicação em bibliotecas acadêmicas. $\mathrm{O}$ instrumento contempla 22 perguntas que cobrem três dimensões das bibliotecas: o espaço, a prestação de serviços e o controle da informação.

- O COUNTER (Counting Online Usage of Networked Eletronic), iniciativa internacional que une bibliotecários, editores e distribuidores de publicações e se apóia em iniciativas e padrões que buscam normalizar as estatísticas de acesso à informação via web.

No Brasil, a mais conhecida experiência de grande porte aconteceu na Universidade de São Paulo. O Sistema Integrado de Bibliotecas da Universidade de São Paulo 
(Sibi/USP) desenvolveu o Programa de Qualidade de Produtos e Serviços de Informação (PAQ) para ser implementado em sua rede de bibliotecas. Englobou duas metodologias diferentes de avaliação, o SERVQUAL e o LibQUAL $+{ }^{\mathrm{TM}}$, ajustadas às características das bibliotecas. No relatório, entre outras importantes conclusões, destaca-se a importância do conhecimento das necessidades do usuário:

No ambiente acadêmico, considera-se que o desconhecimento em relação a essas necessidades pode comprometer as atividades de ensino, pesquisa e a geração de novos conhecimentos (SAMPAIO, 2001, p. 143)

É reconhecido que a Norma ISO 11620 proporciona as condições requeridas para um salto de qualidade no que tange aos processos de avaliação de bibliotecas. A propósito, eis o que ponderou Carbone (1998, p. 41), analisando-a:

Os bibliotecários não podem mais se limitar a relatar seus serviços somente a partir de dados estatísticos sobre os recursos e atividades da biblioteca. Eles devem provar que atendem às necessidades dos usuários e provar de que maneira e a que ponto os serviços da biblioteca as atendem.

A ISO11620 sugere ainda que os resultados da aplicação dos indicadores podem ser comparados em tempos diferentes em uma mesma biblioteca e podem ser feitas comparações entre bibliotecas, ainda que cautela seja sempre necessária. Para o estabelecimento de novos indicadores (conteúdo informativo, comparabilidade, propriedade e outros), devem ser observados critérios, bem como a sua adequada descrição.

\section{O PROJETO}

O projeto Instrumento de Medida de Desempenho para as Bibliotecas do Cict foi iniciado em 2005, com o objetivo principal de propor medidas-padrão para o desempenho das três bibliotecas coordenadas pelo Cict. A equipe diretamente ligada ao seu desenvolvimento é formada por um coordenador, três bibliotecários (um representante de cada biblioteca), dois bolsistas e um consultor.

A etapa inicial envolveu uma busca retrospectiva visando à identificação de relatos de experiências prévias com a utilização da Norma ISO 11620, particularmente no Brasil. No contexto brasileiro, nenhuma referência foi localizada. Os documentos recuperados eram enviados em formato eletrônico para todos os membros da equipe, que se reuniam quinzenalmente para discussões técnicas.
Paralelamente, foi realizado um diagnóstico da situação das três bibliotecas, que procurou explicitar, para cada uma delas o seguinte: o perfil dos usuários; os serviços e produtos oferecidos; a infra-estrutura e recursos humanos disponíveis visando à identificação de fatores relevantes para avaliação. Esse diagnóstico foi de fundamental importância para ressaltar as semelhanças e dessemelhanças entre as três bibliotecas, favorecendo um reconhecimento dos contextos e das práticas individuais.

Esse diagnóstico foi também um pré-requisito para a realização da primeira oficina de trabalho que propiciou a efetiva implantação do projeto, na medida em que esse encontro, incluindo a direção da instituição, foi palco de alianças e "compromissos" primeiros entre os profissionais. A oficina teve como objetivo a discussão coletiva do conjunto de indicadores propostos pela Norma IS011620, e como meta a eleição de um subconjunto dos mesmos para implementação na primeira fase do projeto. Os indicadores foram escolhidos em função do esforço necessário para coleta, consolidação e análise em face do tempo de resposta requerido pelos tomadores de decisão. A eleição dos indicadores levou também em conta a aderência com aqueles já em uso nas bibliotecas, propiciando, assim, uma resposta mais rápida ao planejamento institucional.

Para a dinâmica da oficina, foi adotada a formação de grupos heterogêneos de trabalho no que diz respeito ao desempenho das funções dos participantes, enriquecendo as discussões e compartilhando diferentes visões sobre um mesmo processo. A análise dos indicadores envolveu a conceituação e métricas disponíveis, aplicabilidade e eficácia teórica, oportunidade e propriedade de aplicação no ambiente Fiocruz e possíveis e prováveis adequações necessárias para implementação.

Como resultado foram escolhidos cinco indicadores para aplicação imediata: satisfação do usuário, visitas per capita à biblioteca, índice de uso de documentos, disponibilidade das instalações e índice de ocupação dos assentos. Essa escolha baseou-se no fato de que as metodologias indicadas não apresentariam dificuldades de entendimento e coleta de dados por parte dos profissionais. Evidenciou-se ainda, no curso da oficina, a importância de rever formas de atuação e de adotar mecanismos que viessem a possibilitar melhor desempenho e avaliação de alguns serviços. Ficou clara, também, a importância de uniformizar dados encaminhados para o planejamento central da Fiocruz, responsável pelo orçamento institucional.

Ci. Inf., Brasília, v. 35, n. 3, p. 248-254, set./dez. 2006 
O relatório gerado pela oficina foi chancelado pelas chefias das bibliotecas e encaminhado à direção do Cict. As lacunas e inconsistências foram objeto de atenção na etapa seguinte do projeto.

Dos indicadores selecionados, o primeiro a ser trabalhado foi Satisfação do Usuário, que envolveu a delimitação do público-alvo de cada biblioteca e, em seguida, a identificação dos pontos a incluir no instrumento de coleta. Essa etapa envolveu esforço considerável por parte das bibliotecas, especialmente no que diz respeito às particularidades de cada usuário. Foi desenvolvido um questionário-padrão para coleta de dados, com desenho baseado naquele descrito no Relatório do PAQ (SAMPAIO, 2004).

Em uma escala de quatro níveis, o usuário deveria assinalar o grau de satisfação acerca dos seguintes aspectos das bibliotecas: condições ambientais, qualidade do acervo, serviços oferecidos, atitudes dos profissionais no atendimento ao usuário, qualidade (precisão, atualização e rapidez) da informação prestada e critérios para utilização dos recursos informacionais. Parte do questionário é específica para tratar de aspectos particulares a alguma biblioteca, por exemplo, existência de salão de leitura e acervo de vídeos.

Uma vez elaborado o instrumento, foi realizado um testepiloto em cada uma das bibliotecas, procedendo-se às correções julgadas necessárias. A experiência possibilitou avaliar o impacto do instrumento junto aos usuários, no que tange à sua compreensão e à clareza dos pontos avaliados, bem como identificar lacunas. $\mathrm{Na}$ fase atual do projeto, o instrumento está sendo implementado.

Em paralelo, estão em curso discussões iniciais sobre os outros indicadores selecionados, quais sejam, visitas per capita à biblioteca, índice de uso de documentos, disponibilidade das instalações e índice de ocupação dos assentos, especialmente no que toca à forma de coleta de informações para subsidiar a aplicação dos mesmos.

\section{OS PRÓXIMOS PASSOS}

Confrontadas com os condicionantes técnicocientíficos, socioeconômicos e culturais característicos do final do século XX, as bibliotecas não mais podem retardar a decisão de implantar programas de avaliação de desempenho. Especialmente para as instituições públicas e em face de orçamentos financeiros cada vez mais modestos, conceitos como transparência, responsabilidade, accountability, efetividade e inclusão social/digital colocam aos gestores de bibliotecas desafios nunca antes considerados. O alinhamento com orientações estratégicas institucionais, a necessária priorização na alocação de recursos, o "foco no cliente", a "revolução" das tecnologias de informação e comunicação e a conseqüente virtualização dos acervos e autonomia dos usuários são algumas das perspectivas que tornam imprescindíveis respostas sobre o impacto/ desempenho/valor/qualidade dos serviços das bibliotecas.

Muito se progrediu nas últimas décadas no desenvolvimento de metodologias de avaliação de desempenho. Tal desenvolvimento, entretanto, não camufla a fluidez e subjetividade inerentes ao processo de avaliação e ao conceito de desempenho. De fato, especialistas na área são unânimes em enfatizar que dispor de metodologias adequadas é condição necessária, mas não suficiente para fazer da avaliação um instrumento efetivo e eficaz para a gestão das bibliotecas. Cullen (1997) lembra que décadas de estudos e aprendizado sobre avaliação e dezenas de metodologias robustas disponíveis não substituem o foco principal do sucesso de toda avaliação: liderança profissional e mudança de cultura organizacional. Outros autores vão mais longe (Cramm, 1999) e alertam que só aquelas bibliotecas que já seguem uma "cultura de avaliação" são capazes de levar adiante uma prática de avaliação de desempenho. Por "cultura de avaliação" entenda-se uma cultura de "prática reflexiva" cotidiana, que independe de metodologias formatadas e está intrinsecamente ligada ao compromisso que liga o profissional à instituição, ao usuário, aos colegas de trabalho e às boas práticas profissionais.

As etapas subseqüentes do presente projeto, já planejadas e organizadas em um cronograma, apontam para a continuidade da pesquisa e um bom termo no desenho de uma metodologia para avaliação de desempenho das bibliotecas. O que o futuro não deixa claro, entretanto, é se o processo de aprendizagem vivenciado até aqui será suficiente para que as bibliotecas espelhem as mudanças esperadas (e defendidas) por muitos.

A clareza sobre essa realidade e o envolvimento ativo dos profissionais bibliotecários no projeto são ingredientes essenciais para motivá-los a levar adiante a bandeira da importância decisiva que cabe aos serviços de informação em um contexto de pesquisa. A partir daí, o conceito de qualidade passará ser (re)construído cotidianamente com o usuário e o tomador de decisão.

Artigo submetido em 13/02/2006 e aceito em 13/03/2007. 


\section{REFERÊNCIAS}

CAMILE. Concerted Action on Management Information for Libraries in Europe. Disponível em: <http://www.staff.dmu.ac.uk/ camile/> Acesso em: 01 ago 2005.

CARBONE, P. Évaluer la performance des bibliothèques : une nouvelle norme. BBF: Paris, 1998, n 6, p. 40-45. Disponível em: < http:// bbf.enssib.fr/sdx/BBF/frontoffice/1998/06/document.xsp?id=bbf-1998$06.0040 .005 / 1998 / 06 / \mathrm{fam}$ - apropos/ apropos $\&$ statutMaitre $=$ non $\&$ statut Fils $=$ non $>$. Acesso em: 30 maio 2005.

CRAMM, J. Six impossible things before breakfast:a multidimensional approach to measuring the value of libraries. In: NORTHUMBRIA INTERNATIONAL CONFERENCE ON PERFORMANCE MEASUREMENT IN LIBRARIES AND INFORMATION SERVICES, 3., 1999. Anais... 1999.

CULLEN, R. Does performance measurement improve organisational effectiveness? A post-modern analysis' in Department of Information and Library Management, University of Northumbria at NewcastleIn: (1998) Proceedings of the 2 nd NORTHUMB RIA INTERNATIONAL CONFERENCE ON PERFORMANCE MEASUREMENT IN LIBRARIES AND INFORMATION SERVICES, 2. 1997. Anais... 1998.

INTERNATIONAL FEDERATION OF LIBRARY ASSOCIATIONS AND INSTITUTIONS. International Guidelines for Performance Measurement in Academic Libraries, 1996. Disponível em: < http:// www.ifla.org/V/saur.htm >. Acesso em: 01 ago 2005.

INTERNATIONAL ORGANIZATION FOR STANDARDIZATION: ISO 9004-2 Guidelines for managing the quality of service activities. Genève: ISO, 1991.

. : ISO 11620/98 Information and documentation - library performance indicators. Genève: ISO; 2003a.
: ISO 2789 Information and documentation - library performance . Genève: ISO, $2003 \mathrm{~b}$.

LANCASTER, F. W. Avaliação de serviços de Biblioteca. Brasília: Briquet de Lemos, 1996.

LIBQUAL $+{ }^{\mathrm{TM}}$. Defining and promoting library service quality. Disponível em: $<$ http://www.libqual.org/About/Information/index.cfm $>$. Acesso em: 29 jul. 2005.

MANDANI, Y.The Linnean Society of London and its library. London: Uppsala University, 2004.

MELO, L. B. Estatísticas e avaliação da qualidade e do desempenho em bibliotecas e serviços de informação: investigações recentes e novos projectos. In: Congresso Nacional de Bibliotecários, Arquivistas e Documentalistas, 8., 2004. Anais... Lisboa: APBAD, 2004. Disponível em: < http://eprints.rclis.org/archive/00004156/01/EstAvalBSI_LM 2004.pdf $>$ Acesso em: 17 jul 2005.

PARASURAMAN, A.; ZEITHAML, V. A.; BERRY, L. L. A conceptual model of service quality and its implications for future research. Journal of Marketing, v. 49, p. 41-50, 1985.

ROZADOS, H. B. F. Uso de indicadores na gestão de recursos de informação. Revista digital de biblioteconomia e ciência da informação, v.3, n.1, p.60-76, 2005. Disponível em: <http://server01.bc.unicamp.br/ seer/ojs/viewarticle.php?id=41\&layout=abstract $>$. Acesso em: 10 jul. 2005.

SAMPAIO, M. I. C. et al. PAQ : Programa de avaliação da qualidade de produtos e serviços de informação:uma experiência no SIBI/USP. Ciência da Informação, v. 33, n. 1. p. 142-148, 2004.

SERVQUAL: measuring service quality in academic libraries. 1997. Disponível em: < http://www.arl.org/newsltr/191/servqual.html >. Acesso em: 29 jul 2005. 